\title{
Stochastic Electric Vehicle Network with Elastic Demand and Environmental Costs
}

\author{
Jie Ma $\mathbb{D},{ }^{1,2}$ Hua Wang $\left(\mathbb{D},{ }^{2,3}\right.$ and Tianpei Tang $\mathbb{C}^{4,5}$ \\ ${ }^{1}$ School of Transportation, Southeast University, Nanjing 120096, China \\ ${ }^{2}$ Department of Civil and Environmental Engineering, National University of Singapore, Singapore 117576 \\ ${ }^{3}$ School of Economics and Management, Tongji University, Shanghai 200092, China \\ ${ }^{4}$ School of Transportation and Civil Engineering, Nantong University, Nantong 226019, China \\ ${ }^{5}$ Business School, University of Shanghai for Science and Technology, Shanghai 200093, China \\ Correspondence should be addressed to Hua Wang; hwang191901@gmail.com
}

Received 9 August 2019; Accepted 2 January 2020; Published 12 February 2020

Guest Editor: Hyangsook Lee

Copyright (C) 2020 Jie Ma et al. This is an open access article distributed under the Creative Commons Attribution License, which permits unrestricted use, distribution, and reproduction in any medium, provided the original work is properly cited.

\begin{abstract}
Along with the increasing number of the electric vehicles (EVs), an urban transportation network with a large number of EVs will come true in the near future. Since many countries encourage EVs due to their environmental-friendly benefits, the environmental costs of vehicles have attracted much attention in recent years. In this paper, besides the environmental costs, we take into account the issues of the stochastic user equilibrium (SUE), the elastic demand (ED), and the driving range of EVs in the network. We propose an SUE with ED (SUEED) problem to consider these issues in the urban transportation network with EVs. An SUEED model is developed. We also propose a method of successive average (MSA) to solve the SUEED problem. The computational feasibility of the algorithm is tested in a large-scale network. Through a comparison analysis, we show the benefits of introducing EVs into the urban transportation network in the SUEED circumstance. Moreover, a sensitivity analysis is conducted to reveal the potential values of EVs against the development of EVs. The results suggest that EVs may help to reduce both the travelers' travel costs and the environmental costs of the entire network.
\end{abstract}

\section{Introduction}

The electric vehicle (EV) is one of the most popular topics in recent years. One of the reasons is that many governments around the world are promoting EVs. For example, France, Germany, and the Netherlands claimed that they would substitute gasoline vehicles (GVs) with EVs in a few decades. By the time of January 2018, more than six developed countries and 18 states in the U.S. had joined the group of promoting EVs. There is no doubt that such a large number of EVs will lead to a revolution in the driving behavior. A considerable market penetration rate of EVs is thus considered in this paper. In the literature, an urban transportation network where a large number of EVs and GVs exist simultaneously is referred to as the EV network $[1,2]$.

Besides the governmental promotions, EVs are becoming more and more popular for many reasons. For example, compared with GVs, EVs show their benefits in reducing operation costs, maintenance costs, and fuel consumption, of which the former two are concerned by the drivers and the others are concerned by the governments and the public. Many works have paid attention to these benefits of EVs [1-4]. For example, Jiang and Xie proposed a generalized travel cost function that considers the travel time costs and the operating costs of EVs [2]. Besides these costs, the vehicles' environmental impacts are drawing much attention recently [5-9]. Some works focused on the environmental impacts on special vehicles and carriers $[10,11]$. However, very few works analyze the environmental costs of the private vehicles. A recent study takes into account the environmental costs in the EV network and provides promising results [1]. Through a sensitivity analysis, it suggests that the drivers prefer EVs if they care about the environment or if the 
government improves people's environmental awareness and alleviates EVs' environmental costs. Through a comparison analysis, it suggests that introducing EVs into urban transportation networks can reduce the generalized travel costs for both $\mathrm{EV}$ drivers and $\mathrm{GV}$ drivers.

The implementation of EVs into an urban transportation network would affect travelers' behaviors and in turn the traffic assignment flow pattern which is vital for traffic planning and management. Although EVs have many advantages, the driving range of EVs is much smaller than that of GVs, which may lead to a difference in the driving behavior and the route choice behavior of the travelers. Moreover, the stochastic user equilibrium (SUE) route choice behavior and the elastic demand (ED) are frequently taken into account in the conventional transportation network to build up a realistic traffic assignment model. Although these aspects greatly influence drivers' travel behavior and affect people's choice of EVs to some extent, they are not well considered in the EV network or multimodal networks $[12,13]$. A recent work takes into account the stochasticity of travelers' perception of the generalized travel costs [1]. However, it does not consider the variable travel demand and the limited driving range of EVs, which may lead to an unrealistic flow pattern.

We thus focus on the problem of the driving range of EVs and the elastic demand for the EV network in this paper. The contributions of this paper are listed as follows:

(i) We develop a formulation to consider the environmental cost, the driving range, the $\mathrm{ED}$, and the SUE route choice behavior simultaneously in the EV network. To achieve this, the theory of urban transportation network is employed. We propose a logit-based SUE with ED (SUEED) model for the EV network. In this model, the travelers' environmental cost and route choice behavior are fully considered. The equivalence, existence, and uniqueness of the solution of the proposed model are demonstrated. This model can be easily extended to incorporate other logit-based models, such as C-logit and nested-logit.

(ii) We propose an algorithm to solve the SUEED problem. The original method of successive average (MSA) is revised for the proposed model. With the proposed algorithm, the solution of the proposed model can be guaranteed, and the SUEED problem can be addressed.

(iii) We test the computational feasibility with a largescale network to show the satisfactory computational efficiency of the proposed algorithm. A comparison analysis is conducted to illustrate the impacts of the introduction of EVs and EVs' distance limit, namely, the driving range of EVs, on travelers' choice behavior. We also conduct a sensitivity analysis to estimate the potential value of EVs along with the growth of EV market.

\section{Methodology}

2.1. Notation. The following notations are used in this paper.

Sets:

$A$ : set of links, where $A=\{a\}$

$I$ : set of the travel modes, where $I=\{i\}, i=e$ denotes electric vehicle trips, and $i=g$ denotes gasoline vehicle trips

$K_{w}$ : set of paths between O-D pairs $w$, where $K_{w}=\{k\}$

$N$ : set of nodes, where $N=\{n\}$

$W$ : set of origin-destination (OD) pair nodes, where $W=\{w\}$

Parameters:

$S_{i}^{w}$ : the expected minimal perceived travel cost of mode $i$ between OD pair $w$

$d_{a}$ : physical length of link $a$

$D$ : distance limit for electric vehicles

$D_{i}^{w}(\cdot)$ : travel demand function of mode $i$ between OD pair $w$

$l_{k}^{w}$ : length of path $k$ between OD pairs $w$, where

$l_{k}^{w}=\sum_{a} d_{a} \delta_{a, k}^{w}$

$q_{i}^{w}$ : travel demand of mode $i$ between O-D pair $w$

$q_{i}^{w}$ : upper bound of travel demand of mode $i$ between OD pair $w$

$\delta_{a, k}^{w}$ : link-path incidence parameter, where $\delta_{a, k}^{w}=1$ if link $a$ belongs to path $k$; otherwise, $\delta_{a, k}^{w}=0$

$\theta_{i}$ : travel cost perception errors of travelers with mode $i$

Variables:

$c_{k, i}^{w}$ : travel cost of mode $i$ on path $k$ between OD pair $w$ $f_{k, i}^{w}$ : path flow of mode $i$ on path $k$ between OD pair $w$ $P_{k, i}^{w}$ : route choice probability for travelers with mode $i$ to choose path $k$ between OD pair $w$

$x_{a, i}$ : link flow of mode $i$ on link $a$

2.2. Problem Statement. In this section, we propose a model to describe our problem in the electric vehicle network. The most concerned problems of EV network in this paper are the effects made by the distance limit of EVs and the property of elastic demand (ED). As we know, the traffic network is a complex nonlinear system. This is because the flow pattern of a traffic network is determined by neither a single traveler nor a single road. It is determined by the choice behavior of all the travelers in the network. That is to say, a slight change in the travelers' route choice behavior and the circumstance of the network will make a big difference to the flow pattern of the network $[14,15]$. Moreover, it is also the reason why the theory of urban traffic network, which is famous for its traffic assignment problems (TAPs), was first proposed in the 1960s [16, 17]. The elastic demand and the distance limit of EVs (which are travelers' behavior and networks' circumstance, respectively) are both not-so-small changes. Thus, we employ the theory of urban traffic network to describe our problem. 
2.2.1. Electric Vehicle Network. Let $G=(N, A)$ denote an urban transportation network with EVs, i.e., the EV network, where $N$ denotes a set of nodes and $A$ denotes a set of links. Let $W$ denote the set of origin-destination (OD) pairs. $K_{w}$ represents the set of paths between OD pair $w \in W$. In the EV network, two travel modes, namely, EV trips and GV trips, are taken into account. We thus let $i \in I=\{e, g\}$ denote a set of travel modes, where $i=e$ represents EV trips and $i=g$ represents GV trips. Accordingly, the travelers are classified into two types, i.e., the travelers with mode $e$ and those with mode $g$. We let $f_{k, i}^{w}$ denote the flow of mode $i$ on path $k$ between OD pair $w$; the traffic flow conservation is given by

$$
\sum_{k} f_{k, i}^{w}=q_{i}^{w}, \quad \forall w, \forall i,
$$

where $q_{i}^{w}$ denotes the travel demand of mode $i$ between OD pair $w$. We let the vector $\mathbf{f}=\left(f_{k, i}^{w}, k \in K_{w}, w \in W, i \in I\right)^{T}$ denote the path flow hereafter.

Many works take into account the vehicle driving range and regard it as the biggest difference between the EVs and GVs $[1,2]$. For instance, considering the fact that the driving range of EVs is much shorter than that of GVs, Jiang and Xie defined a distance limit to describe the limited driving range of EVs [2]. In this paper, we inherit this point of view and improve it. Since the technique of EV manufacturing is advancing rapidly, we will examine both the willingness to drive an $\mathrm{EV}$ and how commuters make their travel decisions in terms of the varying distance limit in the circumstances of SUEED. The distance limit of EVs is given by

$$
\left\{\begin{array}{l}
D \geq l_{k}^{w} \Longrightarrow f_{k, e}^{w} \geq 0, \quad \forall w, \forall k, \\
l_{k}^{w}>D \Longrightarrow f_{k, e}^{w}=0, \quad \forall w, \forall k,
\end{array}\right.
$$

where $D$ denotes the driving range of EVs; $l_{k}^{w}=\sum_{a} \delta_{a, k}^{r s} d_{a}$ denotes the length of path $k$ between OD pair $w ; \delta_{a, k}^{w}$ is the link-path incidence parameter, $\delta_{a, k}^{w}=1$ if link $a$ is on path $k$, and otherwise $\delta_{a, k}^{w}=0 ; d_{a}$ denotes the physical length of link a. equation (2) can be rewritten as the following complementarity condition:

$$
\left(D-l_{k}^{w}\right) f_{k, e}^{w} \geq 0, \quad \forall w, \forall k .
$$

Different from the conventional transportation network where only GVs are considered, both EVs and GVs contribute to traffic congestion in the EV network. Therefore, the traffic flow $x_{a}$ on link $a$ in the EV network is defined as follows:

$$
\begin{aligned}
x_{a} & =\sum_{i} x_{a, i}, \quad \forall a, \\
x_{a, i} & =\sum_{w} \sum_{k} \delta_{a . k}^{w} f_{k, i}^{w}, \quad \forall a, \forall i,
\end{aligned}
$$

where $x_{a, i}$ denotes the link flow of mode $i$ on link $a$. We let the vector $\mathbf{x}=\left(x_{a, i}, a \in A, i \in I\right)^{T}$ denote the link flow hereafter. The Bureau of Public Roads (BPR) function is frequently used to depict the travel time in the conventional transportation network. Accordingly, the BPR function for the EV network is revised as follows:

$$
t_{a}(\mathbf{x})=d_{a}\left(1+\alpha\left(\frac{\sum_{i} x_{a, i}}{C_{a}}\right)^{\beta}\right), \quad \forall a,
$$

where the parameters $\alpha=0.15$ and $\beta=4$ in general; $C_{a}$ denotes the capacity of link $a$.

2.2.2. Environmental Cost. Since the environmental impacts of transportation are gaining much attention among the public and the governments, we take into account the environmental costs of the vehicles in the EV network. The environmental cost was first proposed for the EV network by $\mathrm{Ma}$ et al. [1]. It describes the environmental impacts made by the vehicles in an EV network. Tu et al. later extended the environmental cost to the conventional transportation network [18]. In the EV network, both EVs and GVs produce the environmental cost whose amount depends on the unit environmental cost and the travel distance. Therefore, the environmental cost of mode $i$ on link $a$ can be incorporated into the BPR function:

$$
t_{a, i}^{\prime}(\mathbf{x})=d_{a}\left(1+\alpha\left(\frac{\sum_{i} x_{a, i}}{C_{a}}\right)^{\beta}\right)+d_{a} E_{i}, \quad \forall a, \forall i,
$$

where the second term $d_{a} E_{i}$ on the right-hand side is the environmental cost of mode $i$ on $\operatorname{link} a ; E_{e}$ and $E_{g}$ denote the unit environmental cost of EVs and GVs, respectively. In general, $E_{e}<E_{g}$. This is because the GVs produce environmental cost by burning gasoline which releases more harmful gases, while the environmental cost of EVs is much less because of consuming electricity. Moreover, a large proportion of electricity is produced by clean energy in many countries, such as nuclear energy and wind energy. For example, more than $70 \%$ of electricity in France is produced by nuclear; the United States has the most windgenerated electricity in the world [19].

Note that the travel cost function (6a) is applicable only for the network, not for the travelers. This is because travelers only care about their own benefits. However, the environmental cost is an external cost and does not directly hurt their benefits. That is to say, if the travelers do not have any environmental awareness, the environmental cost means nothing to them. To deal with this problem, we thus define the environmental awareness and the generalized travel cost function for the travelers in the E network:

$$
t_{a, i}^{\prime \prime}(\mathbf{x})=d_{a}\left(1+0.15\left(\frac{\sum_{i} x_{a, i}}{c_{a}}\right)^{4}\right)+d_{a} A E_{i}, \quad \forall a, \forall i,
$$

where $A$ denotes the average level of travelers' environmental awareness in the EV network. An interesting discussion about the unit environmental cost $E_{i}$ and the environmental awareness $A$ can be seen in [1].

In general, there are multiple paths between an OD pair for the travelers to choose. Due to the definition of the generalized travel cost function, the path costs become intuitive and equal to the summation of the costs of the links that consist of the paths. The path cost of mode $i$ on path $k$ between OD pair $w$ is given by 


$$
c_{k, i}^{w}=\sum_{a} \sum_{i} \delta_{a . k}^{w} t_{a, i}^{\prime \prime}(\mathbf{x}), \quad \forall w, \forall k, \forall i
$$

2.2.3. Stochastic User Equilibrium. Many researchers proposed various user equilibrium (UE) and SUE models for the conventional transportation network [20-23]. The SUE model takes into account the stochasticity of travelers' perception of the travel cost. One of the most famous SUE models, named the logit-based SUE model, is revised for the EV network herein. The logit-based SUE model assigns the travelers onto the path sets for an OD pair in terms of the generalized travel costs of these paths. Details of the logitbased SUE model can be found in many works (e.g., Daganzo and Sheffi [20]). However, the original logit-based SUE model cannot be directly applied for the EV network because of the different path alternatives for the EVs and the GVs. Since the distance limit of EVs make some paths unavailable for the EVs, the path set for EVs differs from that for GVs. We thus propose a revision of the original logit-based SUE model for the EV network. Because of the travelers' perception error, the perceived path travel cost is given by

$$
C_{k, i}^{w}=c_{k, i}^{w}+\varepsilon_{k, i}^{w}, \quad \forall w, \forall k, l \in K_{w, i}, \forall i \in I,
$$

where $C_{k, i}^{w}$ denotes the perceived path travel cost and $\varepsilon_{k, i}^{w}$ denotes a random perception error with zero mean and constant variance. We assume that $\varepsilon_{k, i}^{w}$ is independently and identically Gumbel-distributed, and then the route choice behavior follows the Logit model. Thus, the route choice probability is

$$
P_{k, i}^{w}=\frac{\exp \left(-\theta_{i} c_{k, i}^{w}\right)}{\sum_{l} \exp \left(-\theta_{i} c_{l, i}^{w}\right)}, \quad \forall w, \forall k, l \in K_{w, i}, \forall i \in I,
$$

where $P_{k, i}^{w}$ denotes the route choice probability for travelers with mode $i$ to choose path $k$ between OD pair $w$ and $\theta_{i}$ denotes the travel cost perception of the travelers with mode $i$. Equation (9) is a slight revision of the logit-based SUE model. It divides the path sets into the path sets for EVs and those for GVs and calculate the route choice probabilities for EVs and GVs, respectively. Accordingly, the path flow of mode $i$ on path $k$ between OD pair $w$ can be obtained by

$$
f_{k, i}^{w}=q_{i}^{w} P_{k, i}^{w}, \quad \forall w, \forall k \in K_{w, i}, \forall i \in I .
$$

Note that the assumption of independence from irrelevant alternative (IIA) underlies the logit model. Many other logit-based models, such as C-logit and nested-logit, were proposed to relax the IIA assumption [24-28]. Our model can easily incorporate these logit-based models as well, while we choose the logit model as an example for generality.

2.2.4. Elastic Demand. The elastic demand (ED) describes a realistic circumstance where the travelers with mode $i$ between OD pair $w$ make their travel decisions in terms of the satisfaction for mode $i$ between OD pair $w$, i.e.,

$$
q_{i}^{w}=D_{i}^{w}\left(S_{i}^{w}\right) \leq \overline{q_{i}^{w}}, \quad \forall w, \forall i \in I,
$$

where $S_{i}^{w}$ denote the satisfaction for mode $i$ between OD pair $w ; D_{i}^{w}(\cdot)$ is a upper bounded strictly monotone decreasing function with respect to the satisfaction $S_{i}^{w} ; \overline{q_{i}^{w}}$ denotes the upper bound of the function $D_{i}^{w}(\cdot)$. We let the vector $\mathbf{q}=$ $\left(q_{i}^{w}, w \in W, i \in I\right)^{T}$ denote the travel demand hereafter. According to the logit-based SUE model [16, 29], the satisfaction is defined by

$$
S_{i}^{w}=-\frac{1}{\theta_{i}} \ln \sum_{k \in K_{w, i}} \exp \left(-\theta_{i} c_{k, i}^{w}\right), \quad \forall w, \forall i \in I .
$$

When the satisfaction for mode $i$ between OD pair $w$ increases, the amount of travelers (i.e., travel demand) decreases. This relationship is called the ED function. Moreover, equations (9) and (11) constitute the SUEED conditions.

2.3. Stochastic User Equilibrium Model with Elastic Demand for EV Network. After introducing above problem statements, we subsequently propose the SUEED model for the EV network as follows:

EV-SUEED:

$$
\begin{aligned}
\min \mathbf{Z}(\mathbf{f})= & \sum_{i}\left[\sum_{w} \sum_{k} \int_{0}^{f_{k, i}^{w}} c_{k, i}^{w}(\omega) \mathrm{d} \omega-\sum_{w} \int_{0}^{q_{i}^{w}(f)} D_{i}^{w-1}(\omega) \mathrm{d} \omega\right. \\
& +\frac{1}{\theta_{i}} \sum_{w} \sum_{k} f_{k, i}^{w}\left(\ln f_{k, i}^{w}-1\right) \\
& \left.-\frac{1}{\theta_{i}} \sum_{w} q_{i}^{w}(\mathbf{f})\left(\ln q_{i}^{w}(\mathbf{f})-1\right)\right],
\end{aligned}
$$

subject to

$$
\begin{array}{r}
\left(D-l_{k}^{w}\right) f_{k, e}^{w} \geq 0, \quad \forall w, \forall i \in I, \forall k \in K_{w, i}, \\
f_{k, i}^{w} \geq 0, \quad \forall w, \forall i \in I, \forall k \in K_{w, i},
\end{array}
$$

where equation (13) is the flow conservation constraint; equation (14) is the distance limit constraint; equations (15) and (16) are the nonnegative constraints which guarantee the nonnegative flow and demand. One may note that equations (9) and (11), i.e., the SUEED conditions, are not in the above constraints. This is because they have been integrated into the objective function [14]; details can be seen in Section 3.

2.4. Equivalence, Existence, and Uniqueness. Herein, we demonstrate the equivalence, existence, and the uniqueness of the SUEED solution based on the model [EV-SUEED].

\subsubsection{Equivalence}

Proposition 1. Any solutions to the model [EV-SUEED] fulfill the SUEED conditions.

Proof. The Karush-Kuhn-Tucker (KKT) condition for the model [EV-SUEED] implies that 


$$
\begin{array}{ll}
P_{k, i}^{w}=\frac{\exp \left(-\theta_{i} c_{k, i}^{w}\right)}{\sum_{l} \exp \left(-\theta_{i} c_{l, i}^{w}\right)}, & \forall w, \forall k, l \in K_{w, i}, \forall i \in I, \\
\sum_{k} f_{k, i}^{w}=D_{i}^{w}\left(-\frac{1}{\theta_{i}} \ln \sum_{k \in K_{w}} \exp \left(-\theta_{i} c_{k, i}^{w}\right)\right), & \forall w, \forall i \in I, \\
\left(D-l_{k}^{w}\right) f_{k, e}^{w} \geq 0, & \forall w, \forall i \in I, \forall k \in K_{w, i}, \\
f_{k, i}^{w} \geq 0, & \forall w, \forall i \in I, \forall k \in K_{w, i}, \\
\text { for the EV network. } & \alpha_{n}=\frac{2 n}{(n+1)^{2}}, \\
& f_{k, i}^{w,(n+1)}=f_{k, i}^{w,(n)}+\alpha_{n}\left(g_{k, i}^{w,(n+1)}-f_{k, i}^{w,(n)}\right), \\
\text { ueness } & q_{i}^{w,(n+1)}=q_{i}^{w,(n)}+\alpha_{n}\left(v_{i}^{w,(n+1)}-q_{i}^{w,(n)}\right),
\end{array}
$$

which is exactly the SUEED conditions and other constraints for the SUEED problem for the EV network.

\subsubsection{Existence and Uniqueness}

Proposition 2. The model [EV-SUEED] has a unique solution.

Proof. We observe that the objective function of model [EV-SUEED] is strictly convex, and its feasible set is convex and compact. According to the theory of convex programming, the existence and uniqueness of the solution are guaranteed.

\section{Algorithm}

Herein, we modify the method of successive average (MSA) and propose an algorithm, named EV-MSA algorithm, to solve the SUEED problem for the EV network. Moreover, we propose a novel step size sequence to speed up the calculation. The proposed EV-MSA algorithm meets the Blum theorem to ensure the convergence [30].

Step 1 (initialization): generate the sets of efficient paths with existing algorithms. Here, we use a K-shortest algorithm proposed by Yen [31] to generate the path set for each OD pair $w$. The path sets are denoted by $K_{e}^{w}$ for electric vehicles and $K_{g}^{w}$ for gasoline vehicles. If the length of a specific path $\widetilde{k}$ is $l_{w}^{w}>D$, we kick it out of $K_{e}^{w}$. Let $\mathbf{x}^{(0)}=0$, calculate the initial free-flow link cost $t_{a}^{(0)}$ and the path cost $c_{k, i}^{w,(0)}$, where $i=e$ for electric vehicles and $i=g$ for gasoline vehicles. Calculate $S_{i}^{w,(0)}$ and $q_{i}^{w,(0)}$ according to equations (12) and (11), respectively. Obtain the initial path flow $f_{k, i}^{w,(0)}$ by equations (9) and (11). Step 2 (update): calculate $x_{a}^{(n+1)}=\sum_{i} \sum_{w} \sum_{k} f_{k, i}^{w,(n)}$ and update $t_{a}^{(n+1)}, c_{k, i}^{w,(n+1)}$, and $C_{i}^{w,(n+1)}$.

Step 3 (finding the search direction): according to $S_{i}^{w,(n+1)}$ obtained in step 2, we calculate the auxiliary demand $v_{i}^{w,(n+1)}$ and then the auxiliary path flow $g_{k, i}^{w,(n+1)}$ according to equations (11) and (9), respectively.

Step 4 (moving): calculate the travel demand and path flows as follows: where $\alpha_{n}=(2 n) /(n+1)^{2}$ is a proposed step size sequence which speeds up the calculation.

Step 5 (convergence criterion): if

$$
\frac{\sqrt{\sum_{i} \sum_{w} \sum_{k}\left(f_{k, i}^{w,(n+1)}-f_{k, i}^{r s,(n)}\right)^{2}}}{\sum_{i} \sum_{w} \sum_{k} f_{k, i}^{w,(n)}} \leq \varepsilon
$$

where $\varepsilon$ is a pregiven accuracy, then the algorithm stops and $\left\{f_{k, i}^{w,(n+1)}\right\}$ is the set of equilibrium flow pattern. Otherwise, return to step 2 .

To verify the proposed model and test the computational efficiency of the proposed algorithm, we conduct a numerical experiment in a large-scale network in Section 4.

\section{Numerical Examples}

In this section, we illustrate the proposed model and algorithm through a large-scale network. The computational feasibility of the algorithm is tested. We also conduct a comparison analysis and a sensitivity analysis in this section.

4.1. Computational Feasibility. TAPs are, in general, largescale problems that are computationally expensive. We hence use the Sioux-Falls network which is one of the most famous large-scale networks for the numerical experiment. The Sioux-Falls network has 24 nodes, 76 links, and 528 OD pairs. For more details, see Figure 1. To test our problem, we generate 10 paths for each OD pair, which means the notation $\delta_{a, k}^{w}$ actually denotes $5,280 \times 76$ parameters. Moreover, since there are two types of private vehicles, the numbers of variables and parameters double than usual. All of these issues make the scale of this example to become quite large. Thus, we take special care about the computational feasibility and efficiency of the proposed method.

To show that the algorithm is efficient enough, we test our example on an ordinary personal computer with a 


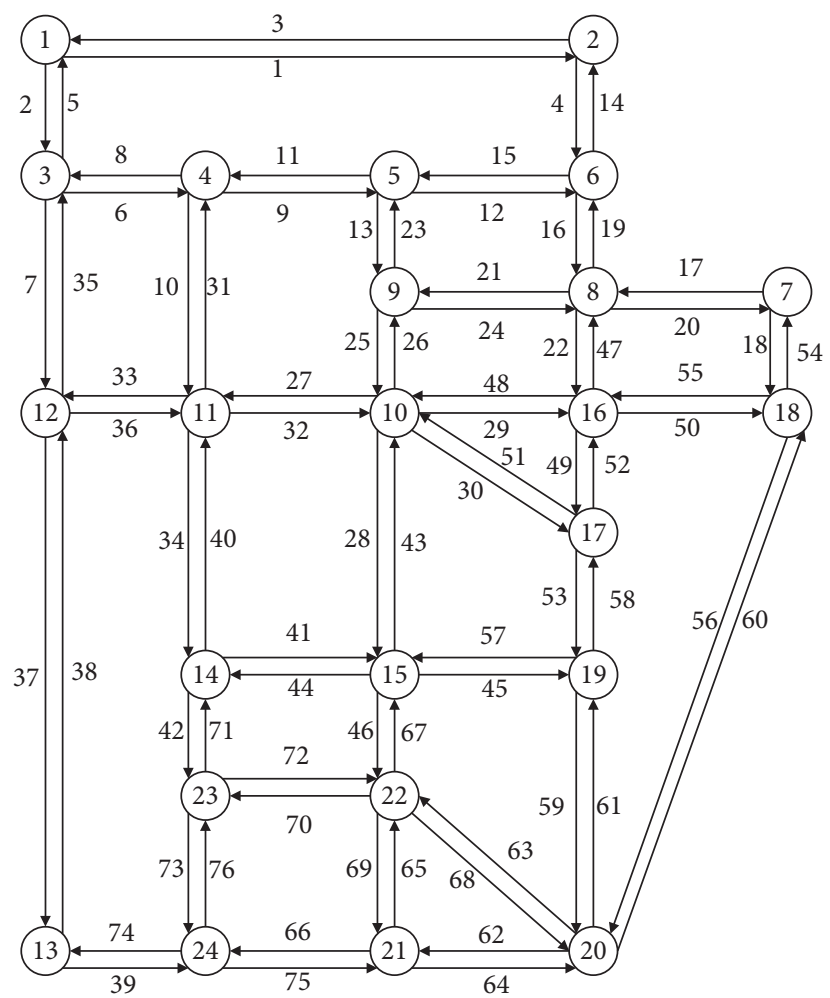

FIgURE 1: Sioux-Falls network.

2.60 GHz CPU, an 8 GB RAM, and Windows 8.1 Enterprise 64-bit operating system.

To build up an elastic demand circumstance for the numerical experiment, any nonincreasing elastic demand functions can be applied. Herein, we use one of the frequently used elastic demand functions as an example:

$$
q_{i}^{w}=D_{i}^{w}\left(S_{i}^{w}\right)=\overline{q_{i}^{w}}-S_{i}^{w}, \quad \forall w, \forall i \in I .
$$

Since many countries have made effort to encourage EVs, the market penetration rate of EVs, namely, the ratio of EVs to private vehicles, will increase to a considerable number in the near future. Hence, we assume a market penetration rate of EVs, whose value can be obtained by survey, in the numerical experiment. The travel demand for EVs and GVs is thus calculated by

$$
\overline{q_{i}^{w}}=\left\{\begin{array}{l}
\lambda \overline{q^{w}}, i=e, \\
(1-\lambda) \overline{q^{w}}, i=g, \forall w
\end{array}\right.
$$

where $\lambda$ denotes the market penetration rate of EVs and $\overline{q^{w}}$ is the total potential travel demand between OD pair $w$. The values of the demand $\overline{q^{w}}$ and the parameters for the BPR function can be found in [32]. We set the accuracy $\varepsilon=10^{-6}$ and the travel cost perception parameters $\theta_{e}=\theta_{g}=0.5$. The algorithm converges to the solution rapidly (see Figure 2 and Table 1).

Figure 2(a) shows the process of convergence. From Figure 2(a) and Table 1, we observe that the algorithm converges quickly. At the $10^{\text {th }}$ iteration, it has already achieved an accuracy of $0.8 \%$. After the $25^{\text {th }}$ iteration, the convergence slows down. Figure 2(b) is a closer look into Figure 2(a). We observe that the whole convergence curve is smooth. No "zig-zag" is observed during the convergence process, which means that the gap between successive intermediate solutions is always getting smaller along with increasing iterations. Figure 2(c) illustrates the variations of the iterations and computational time against the accuracy levels at which the proposed algorithm achieves. The algorithm takes only 0.71 seconds and 213 iterations to converge at an accuracy of $9.9 E-7$.

Table 2 compares the iterations for the MSA and EVMSA to converge at multiple accuracy levels. The results suggest that the proposed step size sequence greatly speeds up the calculation. The proposed EV-MSA is much more efficient than the MSA.

4.2. Comparison Analysis. Besides the computational efficiency, we also care about the impacts of the introduce of EVs and EVs' distance limit on the urban transportation network.

Many works have proved that once the travel demand varies, the flow pattern and, in turn, the generalized travel cost will change nonlinearly $[21,22,33]$. In a former work, we found that the introducing EVs into urban transportation network can reduce the generalized travel costs for both $\mathrm{EV}$ drivers and GV drivers [1]. We are concerned about whether 


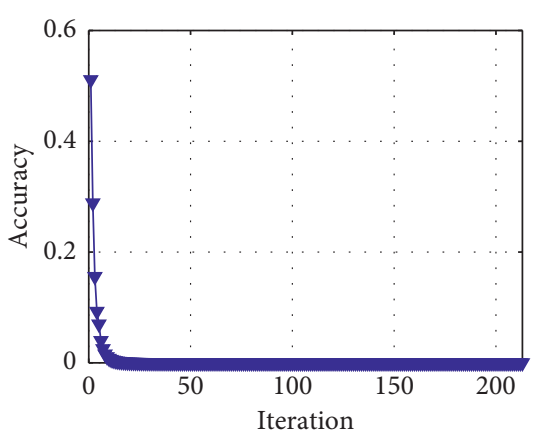

(a)

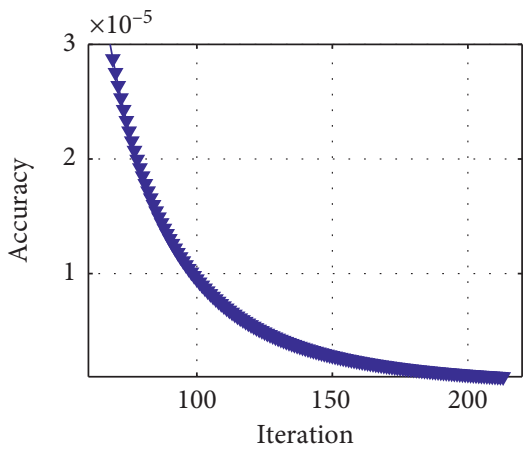

(b)

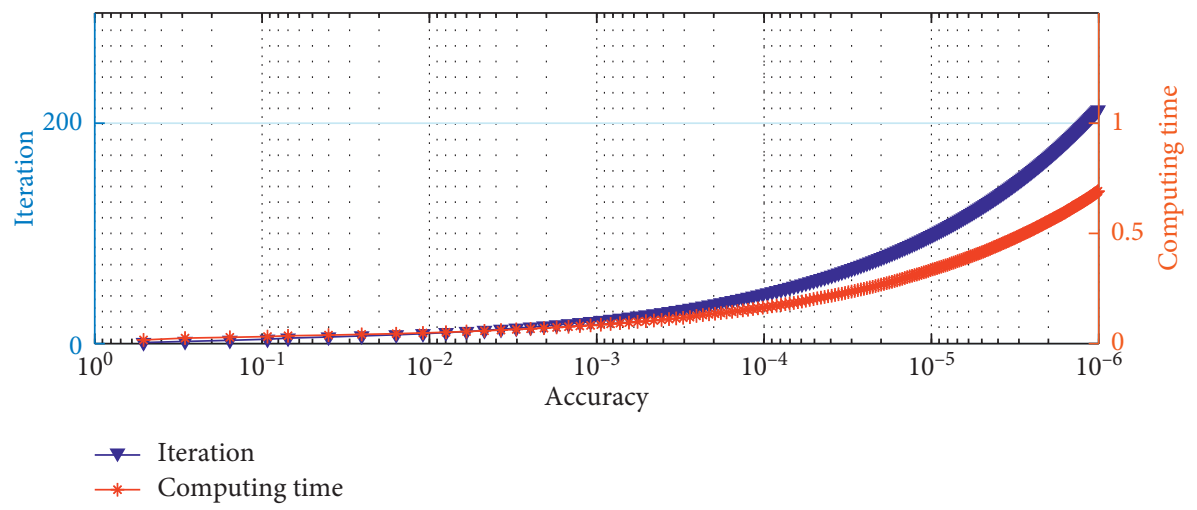

(c)

FIgURE 2: Convergence curves of the EV-MSA algorithm $\left(\varepsilon=10^{-6}\right)$.

TABle 1: Accuracy at specific iterations.

\begin{tabular}{lccccc}
\hline Iteration & Accuracy & Iteration & Accuracy & Iteration & Accuracy \\
\hline 1 & $5.1 E-1$ & 25 & $5.7 E-4$ & 150 & $2.8 E-6$ \\
5 & $7.0 E-2$ & 50 & $7.5 E-5$ & 200 & $1.2 E-6$ \\
10 & $8.0 E-3$ & 100 & $9.5 E-6$ & 213 & $9.9 E-7$ \\
\hline
\end{tabular}

TABle 2: Iterations of MSA and EV-MSA at multiple accuracy levels.

\begin{tabular}{lcccccccc}
\hline Accuracy & $10^{-3}$ & $10^{-4}$ & $10^{-5}$ & $10^{-6}$ & $10^{-7}$ & $10^{-8}$ & $10^{-9}$ & $10^{-10}$ \\
\hline MSA & 44 & 138 & 433 & 1369 & 4338 & 13753 & 43608 & 138268 \\
EV-MSA & 21 & 46 & 99 & 213 & 460 & 990 & 2132 & 4594 \\
\hline
\end{tabular}

this conclusion still holds when the ED and SUE route choice behavior are taken into consideration in the EV network.

Similar to the former work [1], we define the denominator of the right-hand side of equation (9) as the summation of the utilities of all paths between OD pair $w$ for mode $i$, i.e.,

$$
u_{i}^{w}=\sum_{l \in K_{w, i}} \exp \left(-\theta_{i} c_{l, i}^{w}\right), \quad \forall w, \forall i \in I .
$$

We refer to $u_{i}^{w}$ as the utility of mode $i$ between OD pair $w$ hereafter. It can be seen that (a) if the generalized travel cost of a specific path is reduced, the utility will be larger, and (b) in general, the more used paths between OD pair $w$ for mode $i$, the larger utility $u_{i}^{w}$. Therefore, the utility $u_{i}^{w}$ can, to some extent, reflect the service level of mode $i$ for OD pair $w$. Note that, according to the theory of utility maximization $[16,34]$, the satisfaction function $S_{i}^{w}$ is the expectation of the maximum utility alternative (i.e., the path with minimal generalized travel cost) which is strictly monotone with respect to utility $u_{i}^{w}$. Therefore, satisfaction function $S_{i}^{w}$ also reflects the service level of mode $i$ for OD pair $w$. Herein, we use the utility of each OD pair calculated by equation (21). We use $u_{i, 0.8}^{w}$ to represent the utility of mode $i$ for OD pair $w$ in the case that $80 \%$ of vehicles are EVs, and $u_{g, 0}^{w}$ denotes the utility of GVs for OD pair $w$ when no EVs are in the network. The test results can be seen in Figure 3.

For better readability, we rank these OD pairs in terms of a decent order of $u_{g, 0}^{w}$. The dashed, dotted, and solid lines denote the utilities $u_{g, 0}^{w}, u_{g, 0.8}^{w}$, and $u_{e, 0.8}^{w}$, respectively, in a logarithmic coordinate. The bars represent the ratios of the gaps $u_{g, 0.8}^{w}-u_{q, 0}^{w}$ to $u_{q, 0}^{w}$ in a common coordinate. Figure 3 suggests that the utilities for EVs are much higher than those for GVs, which is as expected. This is because EVs have much lower environmental costs than GVs. Besides, we observe that the utilities for GVs are also improved. The 


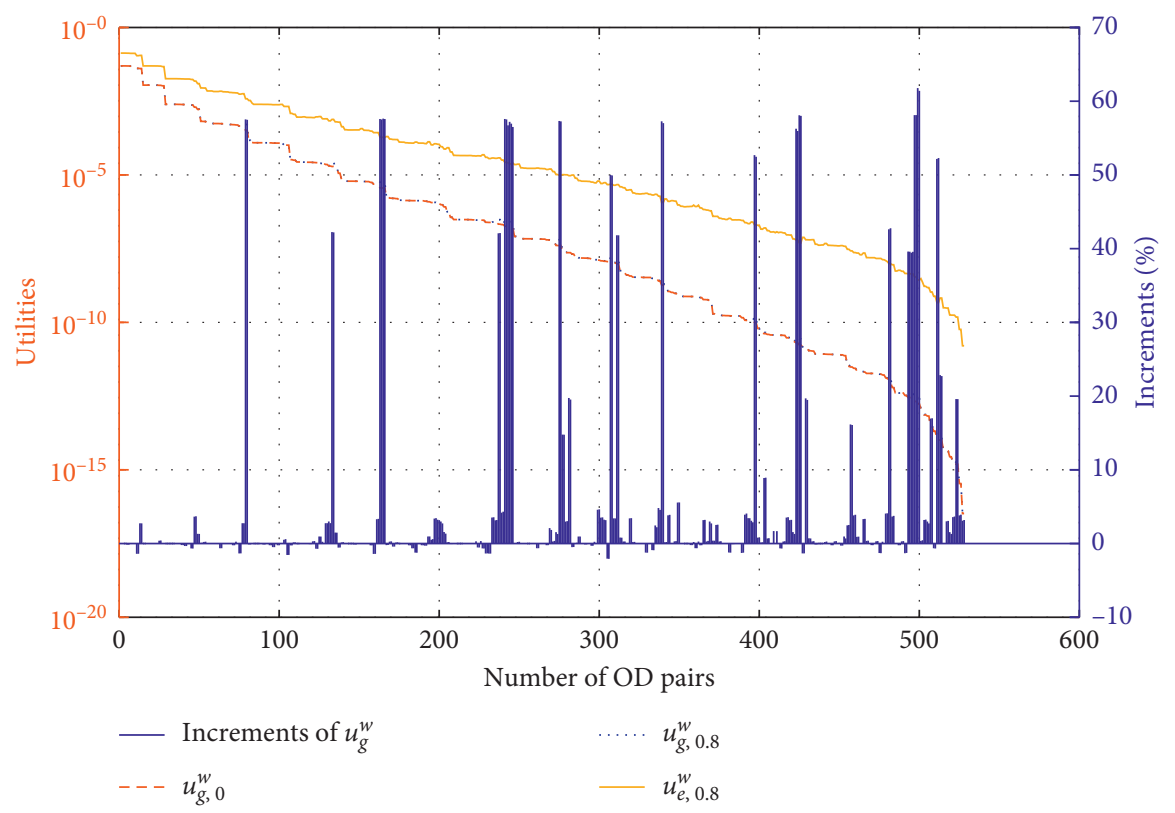

Figure 3: Utilities of 528 OD pairs of Sioux-Falls network with/without EVs.

average increments of GVs' utilities are much higher than the average decrements, and, for most OD pairs, GVs' utilities are higher than before. That is to say, EVs reduce the generalized travel costs for both modes in the network. This conclusion holds when the ED and SUE route choice behavior are considered. This finding provides strong justification to promote EVs because it suggests that EVs are not competitors but cooperators of GVs in reducing travelers' generalized travel costs.

Another issue we are concerned about is the distance limit. Empirically speaking, the battery technique is in a leaptype development. That is to say, the driving range of EVs will be as large as that of GVs in the near future. From this point of view, we conduct a comparison analysis for the cases with and without distance limit. We set the distance limit $D=26$, and let $u_{g, L}^{w}$ and $u_{e, L}^{w}$ denote the utilities for GVs and EVs with distance limit, respectively. The other settings and parameters remain unchanged. The results can be seen in Figure 4.

In Figure 4, the dashed and solid lines represent the utilities for GVs and EVs with distance limit, i.e., $u_{g, L}^{w}$ and $u_{e, L}^{w}$, respectively. Figure 4 shows that, after we remove the distance limit, the increments of the utilities are quite lower compared with those in Figure 3. It suggests that the impact of the distance limit is quite small compared with that of the introduction of EVs. That is to say, although the distance limit is the largest shortcoming of EVs, it cannot deny the benefits that EVs bring to the travelers. Although the driving range limits EVs to a certain degree, introducing EVs into the urban transportation network is still a wise move. Moreover, once the battery industry removes the obstacle of distance limit in the near future, EVs will play a greater role in the urban transportation network.

4.3. Sensitivity Analysis. Different from the leap-type development of battery technique, the market penetration rate of EVs grows step by step. Since some governments plan to ban GVs in the future by law [35], EV's market penetration rate will eventually reach a high level. Hence, we investigate the impacts of EV's market penetration rate on the travelers in the network and see the potential benefits that EVs will bring to the environment. To achieve our goals, we conduct a sensitivity analysis in this section.

We let the market penetration rate $\lambda$ vary from $20 \%$ to $80 \%$ to imitate the growth of EV market. We are concerned about the benefits of EVs for both the travelers and the environment. Moreover, due to the former comparison analysis, we have already known that the influence of the distance limit of EVs is quite small and can be ignored. We hence remove the distance limit when doing the sensitivity analysis for the market penetration rate. The results are illustrated in Figure 5.

The dashed and solid lines represent the total environmental costs $T_{\mathrm{ec}}$ and the total utilities $T_{u}$ of the overall network, respectively, where

$$
\begin{aligned}
T_{\mathrm{ec}} & =\sum_{a} \sum_{i} x_{a, i} d_{a} E_{i}, \\
T_{u} & =\sum_{w} \sum_{i} \sum_{l} \exp \left(-\theta_{i} c_{l, i}^{w}\right) .
\end{aligned}
$$

Note that when calculating the total environmental costs of the network, we use $d_{a} E_{i}$ instead of $d_{a} A E_{i}$ to denote the generalized costs of each link. As mentioned in Section 3 , this is because the environmental cost of the network is a fact that does not depend on people's environmental awareness.

Figure 5 shows that along with the growth of EVs, the total environmental costs decrease and the total utilities keep increasing. It suggests that the growth of EVs can reduce people's generalized travel cost and mitigate harm to the environment. 


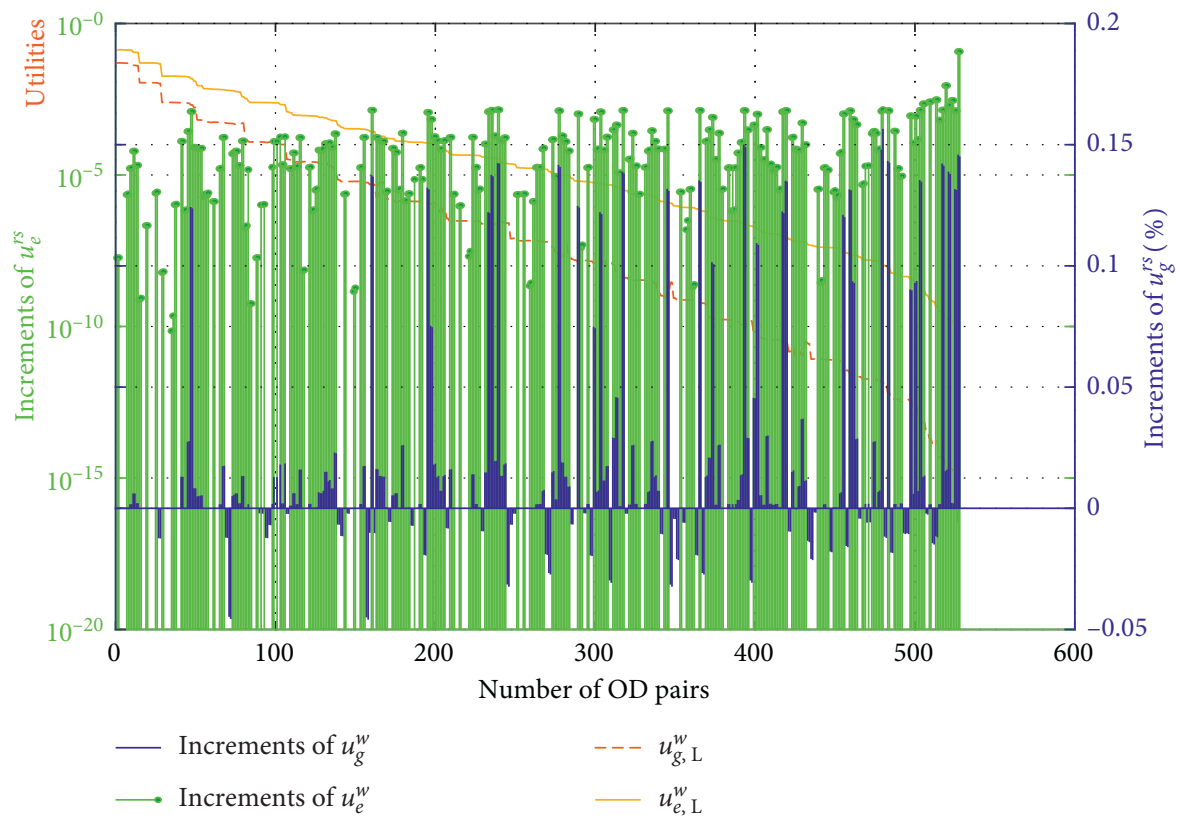

FIgURE 4: Utilities of 528 OD pairs of Sioux-Falls network with/without distance limit.

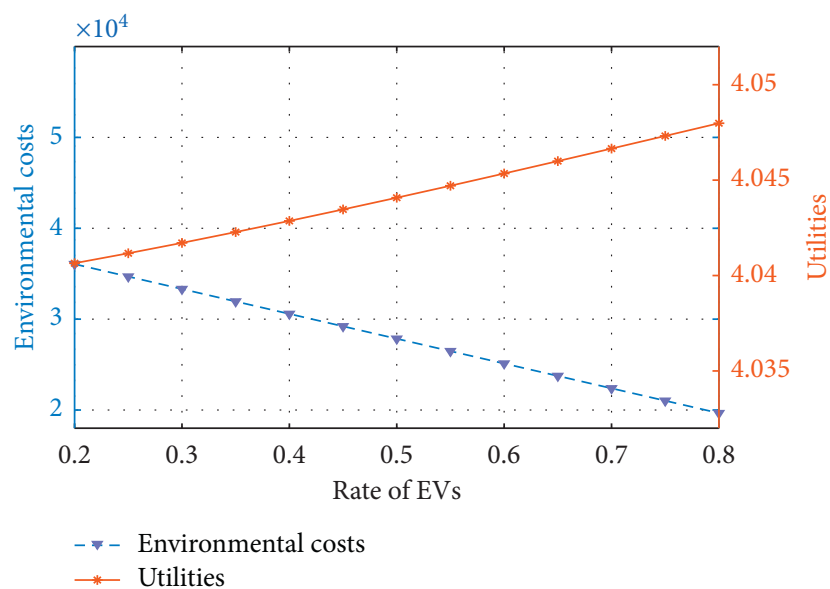

FIGURE 5: Impacts of EV's market penetration rate on environmental costs and utilities.

\section{Conclusions and Future Expectations}

In this paper, we take the environmental cost, EV's driving range, SUE route choice behavior, and ED into consideration and investigate the impacts of EVs on the travelers and the environment. We establish an EV network and propose a generalized travel cost for EVs and GVs in the network. Moreover, a logit-based SUEED model is proposed. By doing these, a more realistic model than existing works for EV networks is established. We also propose an algorithm to solve the proposed SUEED problem in the EV network. Its computational feasibility and efficiency are tested in a largescale network. Through a comparison analysis, we find that introducing EVs into the urban transportation network can reduce the generalized costs for not only EV drivers but also GV drivers in the SUEED circumstance. This finding is interesting because it provides strong justification to promote EVs. Moreover, although the limited driving range of EVs is still a realistic problem, it cannot wipe out the benefits of EVs. Introducing EVs into the network is still a wise move. We conduct a sensitivity analysis to investigate the impacts of the market penetration rate of EVs on the travelers and the environment. We find that, along with the growth of EVs, both the travelers' costs and the environmental costs of the network decrease. It suggests that if we keep promoting EVs as many countries are doing, we will travel in a better circumstance with more satisfaction.

Future challenges may include: (i) the consideration on the uncertainty of the generalized travel costs and the degradability of the EV network; (ii) more travel modes for the travelers to choose; and (iii) the situation that a traveler may possess both EVs and GVs.

\section{Data Availability}

The data used to support the findings of this study are available from the corresponding author upon request.

\section{Conflicts of Interest}

The authors declare that there are no conflicts of interest regarding the publication of this paper.

\section{Authors' Contributions}

J. Ma and $\mathrm{H}$. Wang conceptualized, designed, and analyzed the study and collected data. J. Ma, H. Wang, and T. Tang analyzed and interpreted the results. J. Ma and T. Tang drafted the manuscript. All authors reviewed the results and approved the final version of the manuscript. 


\section{Acknowledgments}

This research was supported by the National Key Research and Development Program of China (no. 2018YFB1600900), the National Natural Science Foundation of China (nos. 71601142, 71971056, and 51608115), the Scientific Research Foundation of the Graduate School of Southeast University (no. YBJJ1840), the Natural Science Foundation of the Jiangsu Higher Education Institutions of China (no. 19KJB580003), the Six Talent Peaks Project in Jiangsu Province (no. XNYQC-003), and the Science and Technology Project of Nantong City (no. JC2019062).

\section{References}

[1] J. Ma, L. Cheng, D. Li, and Q. Tu, "Stochastic electric vehicle network considering environmental costs," Sustainability, vol. 10 , no. 8 , p. $2888,2018$.

[2] N. Jiang and C. Xie, "Computing and analyzing mixed equilibrium network flows with gasoline and electric vehicles," Computer-Aided Civil and Infrastructure Engineering, vol. 29, no. 8, 2014.

[3] Z. Yi, J. Smart, and M. Shirk, "Energy impact evaluation for eco-routing and charging of autonomous electric vehicle fleet: ambient temperature consideration," Transportation Research Part C: Emerging Technologies, vol. 89, pp. 344-363, 2018.

[4] E. Jafari and S. D. Boyles, "Multicriteria stochastic shortest path problem for electric vehicles," Networks and Spatial Economics, vol. 17, no. 3, pp. 1043-1070, 2017.

[5] A. Blackman, F. Alpízar, F. Carlsson, and M. Rivera, "A contingent valuation approach to estimating regulatory costs: Mexico's day without driving Program," Journal of the Association of Environmental and Resource Economists, vol. 5, no. 3, pp. 15-21, 2018.

[6] G. S. Bauer, J. B. Greenblatt, and B. F. Gerke, "Cost, energy, and environmental impact of automated electric taxi fleets in manhattan," Environmental Science \& Technology, vol. 52, no. 8, pp. 4920-4928, 2018.

[7] C. S. Ioakimidis, D. Thomas, P. Rycerski, and K. N. Genikomsakis, "Peak shaving and valley filling of power consumption profile in non-residential buildings using an electric vehicle parking lot," Energy, vol. 148, pp. 148-158, 2018.

[8] Y. Tang, Q. Zhang, Y. Li, G. Wang, and Y. Li, "Recycling mechanisms and policy suggestions for spent electric vehicles' power battery -a case of Beijing," Journal of Cleaner Production, vol. 186, pp. 388-406, 2018.

[9] W. Zhu, D. Yang, and J. Huang, "A hybrid optimization strategy for the maintenance of the wheels of metro vehicles: vehicle turning, wheel re-profiling, and multi-template use," Proceedings of the Institution of Mechanical Engineers, Part F: Journal of Rail and Rapid Transit, vol. 232, no. 3, pp. 832-841, 2018.

[10] C. Mazur, G. Offer, M. Contestabile, and N. Brandon, "Comparing the effects of vehicle automation, policy-making and changed user preferences on the uptake of electric cars and emissions from transport," Sustainability, vol. 10, no. 3, pp. 676-6, 2018.

[11] E. Park, J. Lim, and Y. Cho, "Understanding the emergence and social acceptance of electric vehicles as next-generation models for the automobile industry," Sustainability, vol. 10, no. 3, 2018.
[12] X. Fu and W. H. K. Lam, "Modelling joint activity-travel pattern scheduling problem in multi-modal transit networks," Transportation, vol. 45, no. 1, pp. 23-49, 2018.

[13] C. Sun, L. Cheng, S. Zhu, F. Han, and Z. Chu, "Multi-criteria user equilibrium model considering travel time, travel time reliability and distance," Transportation Research Part D: Transport and Environment, vol. 66, pp. 3-12, 2019.

[14] D. Li, T. Miwa, T. Morikawa, and P. Liu, "Incorporating observed and unobserved heterogeneity in route choice analysis with sampled choice sets," Transportation Research Part C: Emerging Technologies, vol. 67, pp. 31-46, 2016.

[15] D. Li, T. Miwa, C. Xu, and Z. Li, "Non-linear fixed and multilevel random effects of origin-destination specific attributes on route choice behaviour," IET Intelligent Transport Systems, vol. 13, no. 4, pp. 654-660, 2019.

[16] R. D. Luce, Individual Choice Behavior a Theoretical Analysis, Wiley, New York, NY, USA, 1959.

[17] J. Almond, "Traffic assignment with flow-dependent journey times," in Vehicular Traffic Science, pp. 222-234, American Elsevier, New York, NY, USA, 1967.

[18] Q. Tu, L. Cheng, D. Li, J. Ma, and C. Sun, "Stochastic transportation network considering ATIS with the information of environmental cost," Sustainability, vol. 10, no. 11, p. $3861,2018$.

[19] C. P. Barros and S. Managi, "French nuclear electricity plants: productivity and air pollution," Energy Sources, Part B: Economics, Planning, and Policy, vol. 11, no. 8, pp. 718-724, 2016.

[20] C. F. Daganzo and Y. Sheffi, "On stochastic models of traffic assignment," Transportation Science, vol. 11, no. 3, pp. 253-274, 1977.

[21] J. Ma, L. Cheng, and D. Li, "Road maintenance optimization model based on dynamic programming in urban traffic network," Journal of Advanced Transportation, vol. 2018, Article ID 4539324, 11 pages, 2018.

[22] J. Ma, D. Li, L. Cheng, X. Lou, C. Sun, and W. Tang, "Link restriction: methods of testing and avoiding braess paradox in networks considering traffic demands," Journal of Transportation Engineering, Part A: Systems, vol. 144, no. 2, pp. 1-11, 2018.

[23] H. S. Mahmassani and G.-L. Chang, "On boundedly rational user equilibrium in transportation systems," Transportation Science, vol. 21, no. 2, pp. 89-99, 1987.

[24] C. H. Wen and F. S. Koppelman, "The generalized nested logit model," Transportation Research Part B: Methodological, vol. 35, no. 7, pp. 627-641, 2001.

[25] F. S. Koppelman and C. H. Wen, "The paired combinatorial logit model: properties, estimation and application," Transportation Research Part B: Methodological, vol. 34, no. 2, pp. 75-89, 2000.

[26] E. Cascetta, A. Nuzzolo, F. Russo, and A. Vitetta, "A modified logit route choice model overcoming path overlapping problems: specification and some calibration results for interurban networks. Transportation and Traffic Theory," in Proceedings of the 13th International Symposium on Transportation and Traffic Theory, pp. 697-711, July 1996, http:// www.alkox.informatik.hu-berlin.de/lehre/lvws0809/verkehr/ logit.pdf.

[27] M. Bierlaire and E. Frejinger, "Route choice modeling with network-free data," Transportation Research Part C: Emerging Technologies, vol. 16, no. 2, pp. 187-198, 2008.

[28] A. Papola, "Some developments on the cross-nested logit model," Transportation Research Part B: Methodological, vol. 38, no. 9, pp. 833-851, 2004. 
[29] Q. Yu, D. Fang, and W. Du, "Solving the logit-based stochastic user equilibrium problem with elastic demand based on the extended traffic network model," European Journal of Operational Research, vol. 239, no. 1, pp. 112-118, 2014.

[30] J. R. Blum, "Multidimensional stochastic approximation methods," The Annals of Mathematical Statistics, vol. 25, no. 4, pp. 737-744, 1954.

[31] J. Y. Yen, "Finding theKShortest loopless paths in a network," Management Science, vol. 17, no. 11, pp. 712-716, 1971.

[32] H. Bar-Gera, "Transportation networks for research," https:// github.com/bstabler/TransportationNetworks.

[33] Y. Sheffi, Urban Transportation Network, Prentice-Hall, Upper Saddle River, NJ, USA, 1985.

[34] D. McFadden, "Conditional logit analysis of qualitative choice behavior," in Frontiers in Econometrics, pp. 105-142, Academic Press, New York, NY, USA, 1974.

[35] E. R. Wald, "Amsterdam may ban gasoline-powered vehicles in 2030. What will that look like? Forbes," 2019, https://www. forbes.com/sites/ellenrwald/2019/05/07/amsterdam-to-bangasoline-powered-vehicles-in-2030-what-will-that-look-like/ \#652bf39e4214. 